\title{
Computational flow field analysis of a Vertical Axis Wind Turbine
}

\author{
G.Colley $^{1}$, R.Mishra ${ }^{2}$, H.V.Rao ${ }^{3}$ and R.Woolhead ${ }^{4}$ \\ ${ }^{1}$ Department of Engineering \& Technology \\ Huddersfield University \\ Queensgate - Huddersfield, HD1 3DH (UK) \\ Phone/Fax number:+0044 1484 471282, e-mail: g.colley@hud.ac.uk, r.mishra@hud.ac.uk
}

\begin{abstract}
The present work has used Computational Fluid Dynamics (CFD) to obtain the flow field characteristics of a novel Vertical Axis Wind Turbine (VAWT). The turbine used in this study features a multi blade design where both a stator and rotor blade array is used. The computational model is three-dimensional and contains full-scale turbine geometry measuring $2.0 \mathrm{~m}$ in diameter and $1 \mathrm{~m}$ in height. The pressure field across the wind turbine has been computed for operating conditions of $\lambda=0$ and $\lambda=0.4$ where it is shown to be non-uniform. The velocity field across the turbine has been obtained at $\lambda=0$ and $\lambda=0.4$ and has highlighted the presence of a jet flow passing through the central core of the turbine as it exits the rotor assembly. It is evident that under dynamic conditions and due to the direction of this jet flow a strong flow interaction occurs with the downstream rotor blades. This interaction results in rotor blades 5,6 and 7 generating an opposing torque that acts against the direction of motion. Furthermore, it is noticed that rotor blades $10,11,12$ and 1 contribute to the majority of overall rotor torque, which is a characteristic of this machines design.
\end{abstract}

\section{Key words}

Vertical axis wind turbine, CFD, flow field.

\section{Introduction}

Renewable energy technologies play a key role in the contribution to sustainable energy as a whole. Such technologies reduce our dependency on fossil fuel reserves and pave the way for long-term energy security.

Wind power has the potential to contribute significantly towards the sustainable energy sector over the coming years. This technology is seen to be the first renewable power generation technology (excluding large hydro projects) to become a genuine mainstream alternative for increasing the generation capacity across the globe $[\mathbf{1 , 2}]$. Recent wind energy reviews have reported a rise of 22,000 MW in installed generation capacity in Europe between 2004 - 2007. This considerable increase has been the primary factor for global annual growth rates and recent studies state $60 \%$ of global installed capacity is now present in Europe alone [3].

Over a period of 30 years, significant developments have been made in the wind engineering sector. Given the importance of this sector, a considerable amount of research has been carried out on the optimization of traditional turbine designs with a view to improving the energy capture/conversion efficiency. Further to this work, many research groups have proposed novel design configurations again with a view to generating high levels of power.

Upon reviewing the available literature, such machines are being designed without consideration to the installation site and wind conditions present. Many are of the view that if the UK is to continue increasing installed capacity, wind turbine manufacturers should look at installing machines in the urban environment. Due to the considerably lower cost of site, development along with the un-tapped residential market a turbine designed specifically for the urban environment should be investigated.

Given the low wind speeds present in this environment, start-ability is a primary concern. Solutions to this problem are in the form of high solidity multi-blade machines that generate high starting torques at both low wind and rotor speeds. The work of Takao et al [4] presents a novel radial cross flow wind turbine featuring five equally spaced NACA 0015 profile blades. Surrounding the blade inlet zone is a set of outer guide vanes, which are directed into the steam wise flow by a downstream tail vane. The turbine measures $0.6 \mathrm{~m}$ in diameter and $0.7 \mathrm{~m}$ in height with the machines performance output determined from wind tunnel tests. The authors report increases in power output of 1.5 times for the turbine with guide vane row.

Further investigations have been carried out into the effects of varying rotor solidity in which the number of blades is varied from 2-5. The turbine generates a power coefficient of 0.085 at a tip speed ratio of 0.95 for a five bladed rotor whereas reducing the number of blades results in a power coefficient of 0.15 at a tip speed ratio of 1.6. This highlights both the effect of solidity on peak power but also the speed up effects by reducing the number of blades.

Further novel designs have been documented in the form of utility scale vertical axis turbines. Park and Lee et al $[\mathbf{5 , 6 ]}$ have presented a radial cross flow multi blade VAWT that features a set of outer guide vanes again directed by a downstream tail vane. Here the outer guide vanes are placed upstream and are used to accelerate the flow into the rotor blade passages. A secondary side collector is used to funnel the flow into the passages on the leeward side of the machine, which would be otherwise un-used. Such modifications have allowed the authors to maximize power output and have reported 
power coefficients in the order of 0.45 for the baseline six bladed design. The effects of blade number and turbine aspect ratio on power output have also been quantified with the aim of optimizing power coefficient.

The above references provide details of machines that are particularly suited to such a low speed environment. The turbine presented in this work is of similar nature and has been designed specifically for use in the urban envoronment. This machine features a multi blade radial design in which an outer array of guide vanes has been used.

Given the novelty of this design, little information is available on the flow field data within the machine. The present study will analyse both the resultant pressure and velocity fields with a view to determining key energy transfer regions and highlight machine inefficiencies.

\section{CFD Model Design}

The CFD model used in this study features full scale wind turbine geometry and measures $2.0 \mathrm{~m}$ diameter $\mathrm{x}$ $1.0 \mathrm{~m}$ in height. A plan view of the CFD flow domain is depicted in figure 1 .

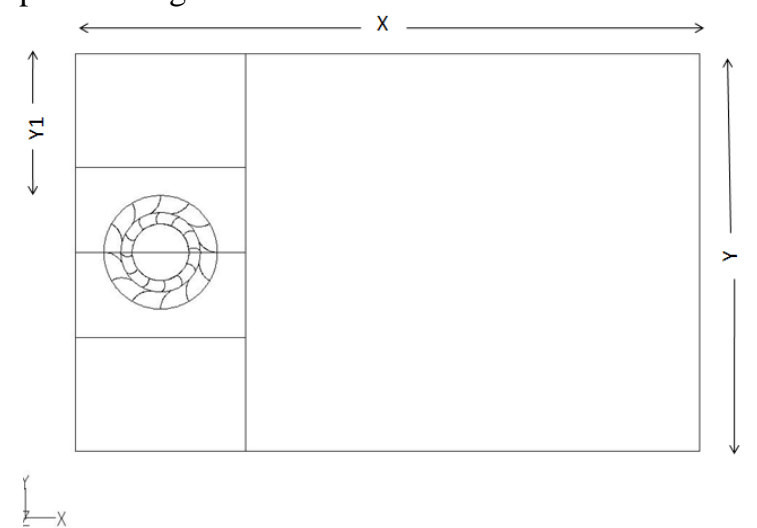

Figure 1. CFD Flow domain in XY plane Where: $X=11 \mathrm{~m}, Y=7 \mathrm{~m}, \mathrm{Y} 1=2.5 \mathrm{~m}$

The location of the wind turbine pictured in the ZX plane is shown in figure 2 . Here the location of the blade geometry relative to the neighbouring boundaries is described.

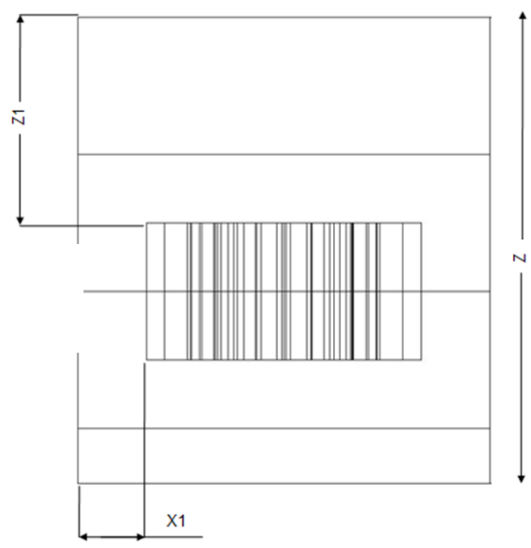

Figure 2. CFD Flow domain in XY plane Where: $\mathrm{Z}=3.4 \mathrm{~m}, \mathrm{Z} 1=1.5 \mathrm{~m}, \mathrm{X} 1=0.5 \mathrm{~m}$
The wind turbine geometry used in this study is shown in detail in figure 3 . This figure highlights the different zones present within the wind turbine along with the machines orientation relative to the free-stream flow direction $(\mathrm{V} \infty)$.

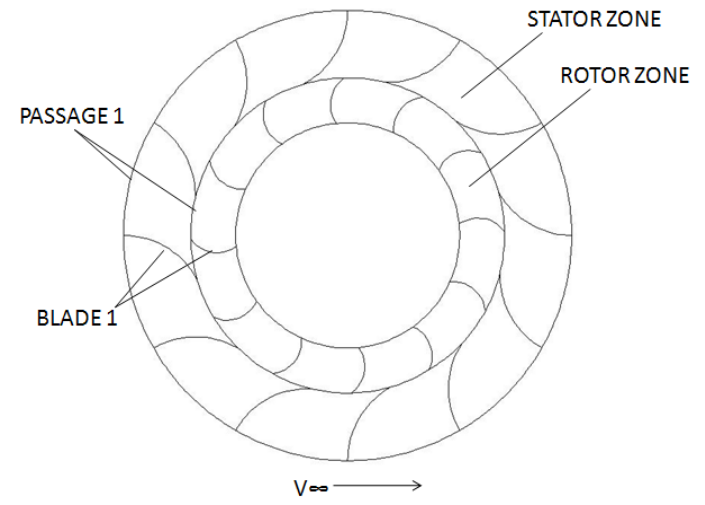

Figure 3. Wind turbine geometry realtive to free-steam flow

\section{CFD Methodology}

In the present work a Multi Reference Frane (MRF) solving technique has been used to carry out a series of steady state simulations at a fixed rotor position. This corresponds to the position at which rotor mean torque is generated as identified from preliminary studies. In this case, two separate fluid rings have been created around the stator and rotor blades. The fluid continuum corresponding to the rotor zone is rotated relative to both the stationary rotor blades and the stationary stator fluid zone. It is this rotation of fluid against the stationary blades that is used to compute the forces acting on the rotor and hence the reaction given from the rotor to the fluid.

The meshing strategy used in this work uses both structured and un-structured type grids. The use of the structured grid is limited to the outer regions of the rectangular flow domain where the boundary geometry is simple. A tetrahedral type mesh has been applied to the wind turbine blade geometry due to the complex nature of its design. The total number of mesh elements used in this study is approximately 3.2 million. Due to the demanding computational requirements, the grid has been partitioned and distributed over a multi core linux based cluster.

The boundary conditions used during this set of simulations correspond to those present in the laboratory area where experimental testing takes place. The realizable $\mathrm{k}-\varepsilon$ viscous solver [7] has been used in the following simulations due to its stability during computation and accuracy over a wide range of operating conditions. The influx of air into the flow domain is governed by the velocity inlet boundary at which a uniform $4 \mathrm{~m} / \mathrm{s}$ flow condition is used in the X-direction. The velocity inlet is located $0.5 \mathrm{~m}$ away from the turbine and spans over the entire turbine inlet. 


\section{Results}

In the present work, the flow field characteristics across the wind turbine are investigated for both a static and dynamic rotor condition. These operational conditions correspond to rotor tip speed ratios $(\lambda)$ of 0 and 0.4 respectively. The rotor blade tip speed is defined in nondimensional form relative to the free-stream velocity $(\mathrm{V} \infty)$ as per the following equation where $\lambda$ represents the rotor blade tip speed ratio. Here, the radius of the rotor tip (r) is taken to be $0.7 \mathrm{~m}$.

$$
\lambda=\frac{\omega \cdot r}{\mathrm{~V} \infty}
$$

\section{Pressure Field Analysis}

In the following the static pressure fields for $\lambda=0$ and $\lambda 0.4$ have been computed and are depicted in figures 4 and 5. The turbine blade number used in this analysis is defined by figure 3 where blade number increases from 1 to 12 in the clockwise direction.

This pressure field shows similarities between both operating conditions in which the pressure is nonuniform about the central axis of the machine. Further observations are the high pressure regions present over stator blades 12 and 1 , which again are consistent between both conditions.

Upon further observation, subtl variations in the pressure field within the rotor fluid ring are present. During the static rotor condition three regions of negative pressure are present over rotor blades 11,12 and 1. This is due to the rotor blade position relative to the neighbouring stator blades. In this region a reduction in cross sectional passage area results in local flow accelerations towards the inlet of the rotor. It is seen at $\lambda=0.4$ this pressure increases to above or equal to atmospheric pressure.

The pressure field for $\lambda=0.4$ shows almost identical characteristics over the turbine and is of similar magnitude. The only variation here is the increase in pressure at rotor blade positons 11,12 and 1 .

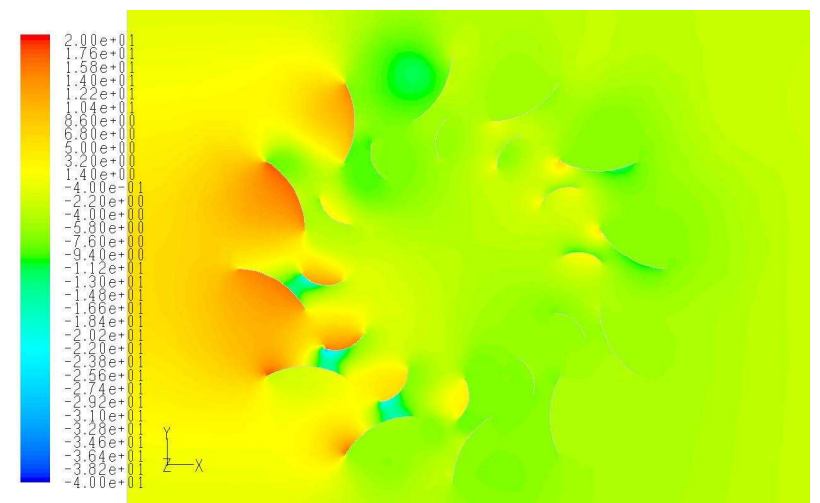

Figure 4. Static pressure field at turbine midplane for $\mathrm{V} \infty=4 \mathrm{~m} / \mathrm{s}$ and $\lambda=0$

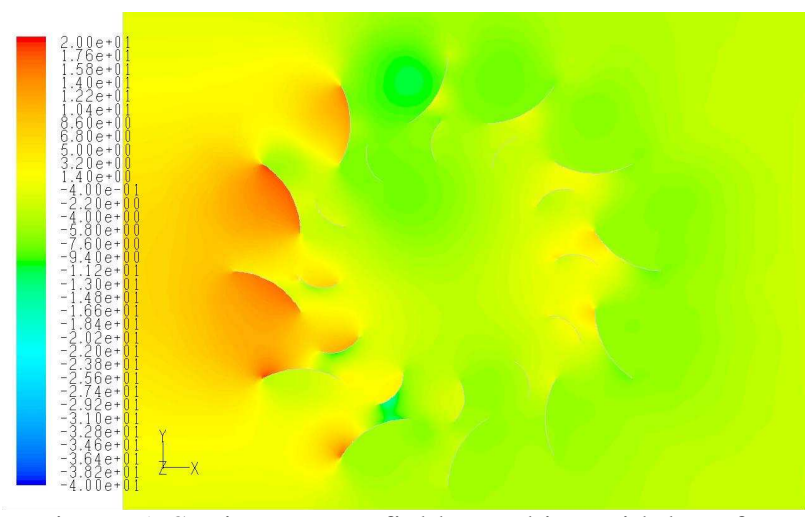

Figure 5. Static pressure field at turbine midplane for $\mathrm{V} \infty=4 \mathrm{~m} / \mathrm{s}$ and $\lambda=0.4$

To understand the variation in pressure at each of the blade passage inlet/outlet boundaries, the area weighted average pressure has been computed over each face. This pressure has been computed at the stator inlet, rotor inlet and rotor outlet face boundaries for each blade passage. The area weighted pressure is plotted against blade passage number as per figure 3 . In each of the plots the passage pressure is plotted for both the static and dynamic rotor conditions.

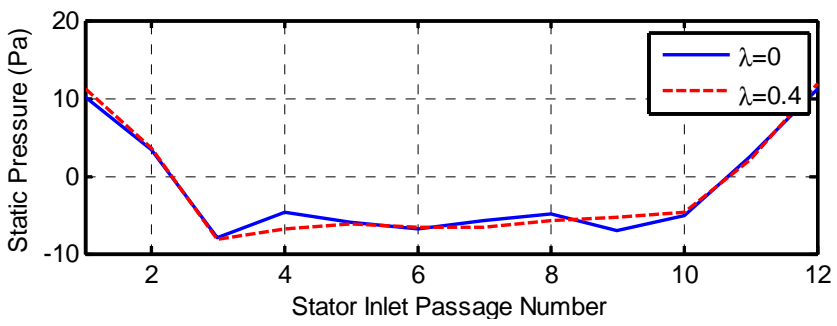

Figure 6. Area weighted average static pressure computed over stator passage inlet faces at $\mathrm{V} \propto=4 \mathrm{~m} / \mathrm{s}$ for $\lambda=0$ and $\lambda=0.4$

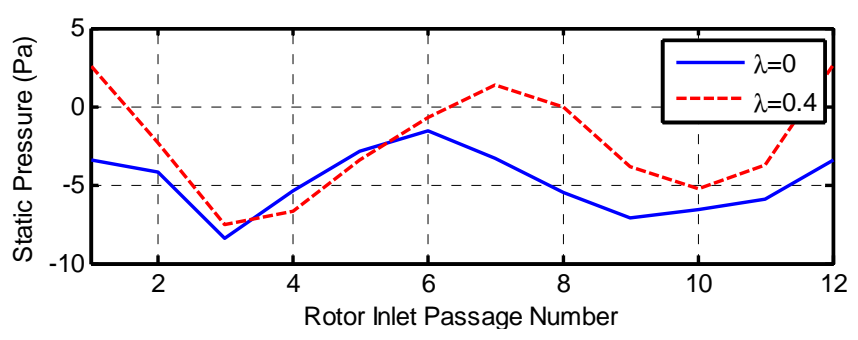

Figure 7. Area weighted average static pressure computed over rotor passage inlet faces at $\mathrm{V} \propto=4 \mathrm{~m} / \mathrm{s}$ for $\lambda=0$ and $\lambda=0.4$

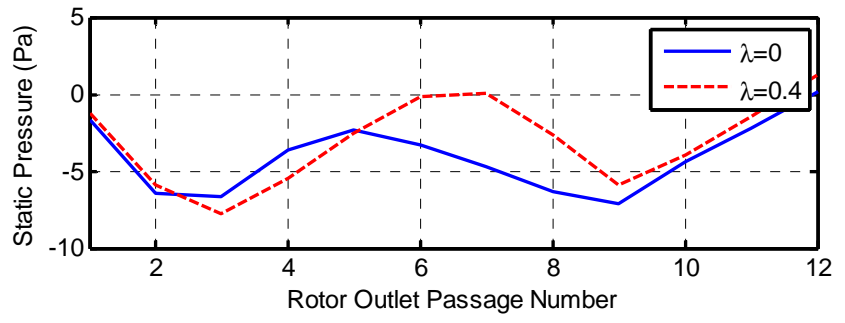

Figure 8. Area weighted average static pressure computed over rotor passage outlet faces at $\mathrm{V} \propto=4 \mathrm{~m} / \mathrm{s}$ for $\lambda=0$ and $\lambda=0.4$ 
Figure 6 depicts this pressure variation at the stator blade passage inlet boundaries for both static and dynamic conditions. It is noticed that minimal variation occurs between each operating condition with mangitudes of pressure being similar throughout. Notable observations include the large region of negative pressure between passages 3 and 10 for both $\lambda=0$ and $\lambda=0.4$. Maximum pressures are present at the inlets of passages 1,2,11 and 12 , which is to be expected due to the orientation of the blades relative to the free-stream flow direction.

A similar analysis has been carried out to determine the magnitudes of pressure across the rotor passage inlet faces. The pressure distribution across these boundaries governs the energy tranfer process within the machine and hence is of particular importance. Figure 7 shows this pressure distribution for both $\lambda=0$ and $\lambda=0.4$. Again as with the stator inlet face distribution a noticable trend is present which shows similarities between $\lambda=0$ and $\lambda=0.4$. A notable observation is the increase in pressure at almost all inlet faces when the rotor is in motion.

Similar trends are seen when observing the pressure distribution at the exit of the rotor blade passages as shown in figure 8 . Here an almost identical trend is present in which the pressure increases at almost all passage exit faces when the rotor is in motion.

\section{Velocity Field Analysis}

The resultant velocity field generated across the machine for both static and dynamic rotor conditions is depicted in figures 9 and 10. The nature of the flow field within the machine is clearly visible with some interesting flow features being generated. One notable feature is the jet of air passing through the central core of the turbine. The direction of the jet at $\lambda=0$ clearly influenced by the orientation of the windward static rotor blades. This orientation re-directs the free-sream flow in an anti clockwise shift as it exits the stationary rotor assembly.

The effect of rotor motion on the turbine velocity field is presented in figure 10. The velocity field shown here contains many similarities to that shown for $\lambda=0$. The velocity mangitude and localised distributuon within the stator blade passages is almost identical which indicates weak interaction effects between the rotor fluid ring and the surrounding stator zone. The velocity field within the rotor fluid ring and central core of the machine shows significant differences to that presented at $\lambda=0$. The jet of air passing through the machine is re-orientated with the stream-wise flow direction as it enters and exits the rotating rotor assembly, which may result in downstream interaction effects with the rotor blades.

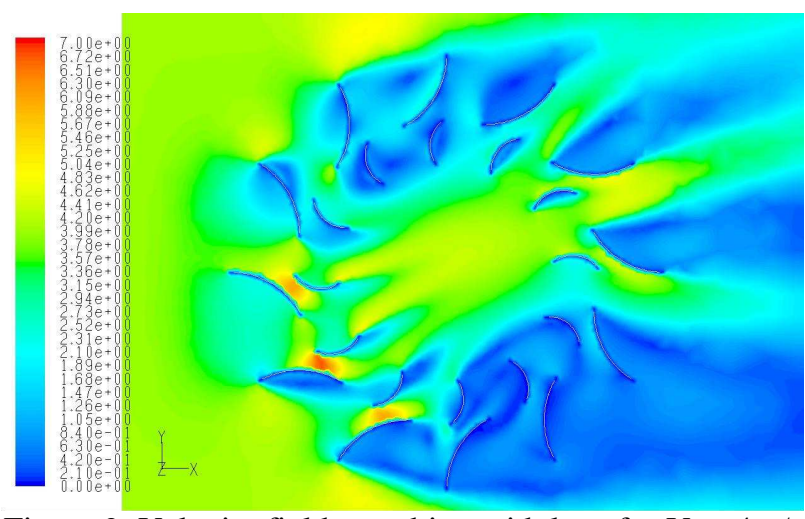

Figure 9. Velocity field at turbine midplane for $\mathrm{V} \infty=4 \mathrm{~m} / \mathrm{s}$ and $\lambda=0$

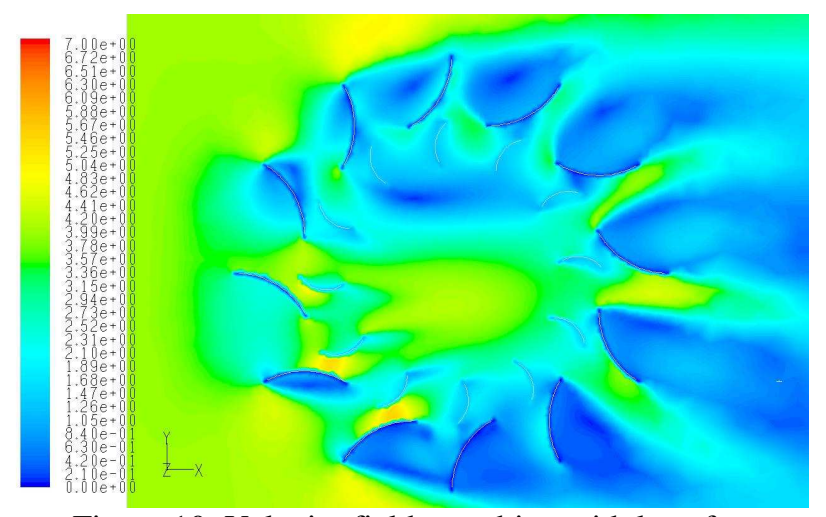

Figure 10. Velocity field at turbine midplane for $\mathrm{V} \infty=4 \mathrm{~m} / \mathrm{s}$ and $\lambda=0.4$

In order to understand the nature of the flow features across the machine, a detailed plot of velocity vectors is provided in figures 11 and 12 . The magnitude of velocity governed by the length of the arrow along with its direction shows how the pressure and velocity fields govern the flow structures present within the machine. This is particularly visible in how the pressure and velocity fields dictate the direction of flow across the blades and ultimately control the energy transfer process.

The influence of the rotor under dynamic conditions is again apparant due to the change in flow structures within the core of the machine. In both static and dynamic conditions a main jet of air can be seen as it enters the windward stator passages and passes through the rotor assembly. At an operating condition of $\lambda=0.4$ the direction on this jet of air is seen to shift in the clockwise direction as per figure 12 and further validates the conclusions drawn from figure 10.

This directional shift results in the development of a vortex ring in the core of the turbine, which has a relatively low velocity. The generation of this vortex ring causes a change in the region where energy transfer takes place as it shifts to the opposing side of the rotor. This region is defined by the dashed ring in figure 12 and highlights the significant contribution of a few key blades to overall rotor torque. 


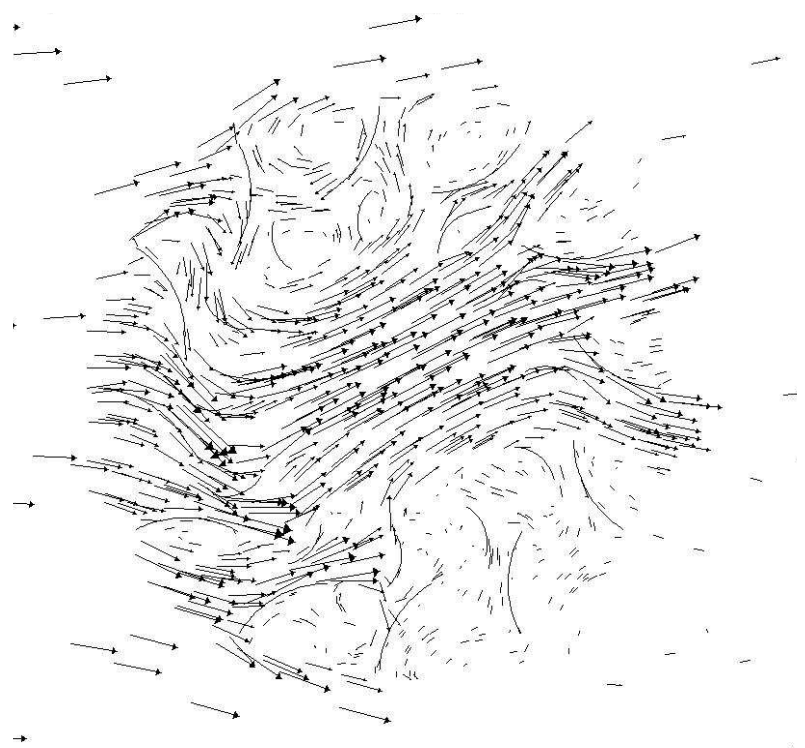

Figure 11. Velocity vector field at turbine midplane for $\mathrm{V} \infty=4 \mathrm{~m} / \mathrm{s}$ and $\lambda=0$

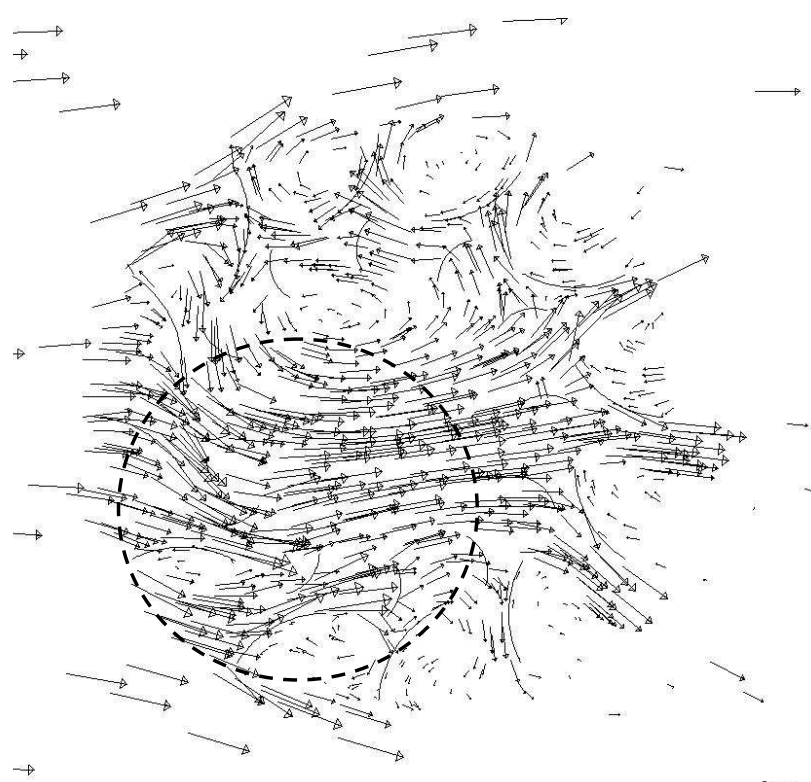

Figure 12. Velocity vector field at turbine midplane for $\mathrm{V} \infty=4 \mathrm{~m} / \mathrm{s}$ and $\lambda=0.4$

In both rotor operating conditions the main jet of air passing through the machine interacts with the leeward side rotor blades as it exits the assembly. Such interaction effect are the primary cause for opposing torque generation with this type of turbine as highlighted in previous studies.

To understand the result of such interaction effects, the individual torque contribution of each rotor blade is computed. This torque output is plotted in nondimensional form of $\mathrm{T} / \mathrm{T}_{\mathrm{MAX}}$ where $\mathrm{T}_{\mathrm{MAX}}$ corresponds to total rotor torque output at a given operating condition. Figure 13 depicts this torque output for each of the rotor blades.

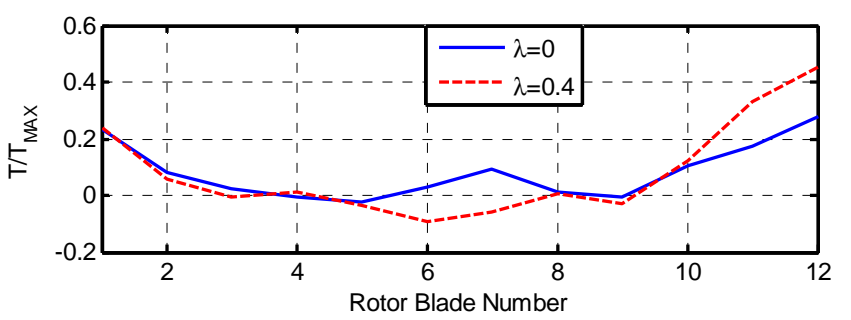

Figure 13. Rotor blade non-dimensional torque output at $\mathrm{V} \propto=4 \mathrm{~m} / \mathrm{s}$ for $\lambda=0$ and $\lambda=0.4$

The indivdual rotor blade torque output shows significant variation between operating conditions of $\lambda=0$ and $\lambda=0.4$. The torque output generated at $\lambda=0$ is seen to be positive for almost all rotor blades. At this static condition rotor blade 5 generates a negative torque, which opposes the direction of motion. The torque output for blades 3-9 is seen to be minimal with the exception of blade 7 . Maximum torques are generated by blades $1,2,10,11$ and 12 which is due to their orientation relative to the flow.

Rotor blade torque output computed for $\lambda=0.4$ shows some variations when compared to the static condition. The torque output generated by rotor blades 10,11 and 12 is significantly higher which further validates the conclusions drawn from the plots of velocity vectors at this condition. Furthermore, the torque output for blades 6 and 7 is considerably higher and still acts against the direction of motion. Such effects are a result of the fixed blade design and downstream interaction with the jet of air. This characteristic is well documented and is present for the majoirty of vertical axis designs. Given the work carried out in this study and the identification of such phenomena, further work should be dedicated to conditioning the central jet of air to avoid downstream blade interaction. By minimzing such interaction effects the energy capture/conversion efficiency can be increased considerably.

\section{Conclusions}

In the following the main conclusions from this study have been summarised:

- The pressure field across the wind turbine has been computed for operating conditions of $\lambda=0$ and $\lambda=0.4$. It has been shown to be non-uniform about the central rotational axis of the machine.

- The area-weighted static pressure has been computed at each blade passage inlet and outlet face and has identified the regions where maximum energy transfer takes place.

-The effect of rotor speed on the above pressure fields has been quantified and is shown have minimum effect on the surrounding stator zone. This characterstic is a result of the weak interaction effects between the rotor fluid ring and stator zone coupled with the use of the MRF solver. 
-The velocity field across the turbine has been obtained at $\lambda=0$ and $\lambda=0.4$. This has highlighted the presence of a jet flow passing through the central core of the turbine as it exits the rotor assembly.

-The effect of rotor speed on this jet flow is shown to have significant effects in terms of direction. At $\lambda=0$ an anti-clockwise shift from the streamwise direction is noticed. At condition $\lambda=0.4$ the rotor causes a clockwise shift back towards the stream-wise flow direction.

-The shift in jet flow direction under dynamic coniditons results in the formation of a central vortex ring in the core of the machine. This formation shifts the primary energy transfer region to the opposing side of the machine.

-This energy transfer shift is validated by an increase in blade torque contribution in this area in which a small number of rotor blade contribute significantly to overall turbine torque.

-It is evident that under dyanmic conditions and due to the direction of the jet flow a strong interaction occurs with the downstream rotor blades.

-This interaction results in rotor blades 5,6 and 7 generating an opposing torque that acts against the direction of motion.

\section{Nomenclature}

$\mathrm{V} \infty=$ Free-stream velocity $(\mathrm{m} / \mathrm{s})$

$\mathrm{P}=$ StaticPressure $(\mathrm{Pa})$

$\lambda=$ Tip Speed Ratio

$\omega=$ Angular Velocity ( $\mathrm{rad} / \mathrm{s})$

$\mathrm{r}=$ Rotor radius $(\mathrm{m})$

$\mathrm{T}=$ Torque $(\mathrm{Nm})$

$\mathrm{T}_{\mathrm{MAX}}=$ Total rotor torque $(\mathrm{Nm})$

\section{References}

[1] EWEC, Wind energy review. (2009)

[2] BWEA, England's Regional Renewable Energy Targets:

Progress report. (2009)

[3] EREC, Renewable energy framework directive. (2007)

[4] M.Takao. A straight bladed vertical axis wind turbine with a directed guide vane row. Thermal Science Vol.18, No.1 (2009) 54-57

[5] J. Park, S. Lee, T. Sabourin and K. Park, A novel Vertical Axis Wind Turbine for Distributed \& Utility Deployment. Ontario Sustainable Energy Association, (2007)

[6] S. Lee, W. Song, J. Park and Y.Kim, Experimental study on control performance of jet wheel turbo wind turbine. Int Conference on Fluid Machinery, (2007), Korea
[7] A.Gosman, Developments in CFD for industrial and environmental application in wind engineering. J.Wind Eng. Ind. Aerodyn. 81 (1999) 21-39. 